cervix, and $18.3 \%$ were both local and systemic IgA antibody negative.

Conclusion After the third vaccination, there is a strong agreement between cervical and systemic IgG antibody responses and a weak agreement between cervical and systemic IgA antibody responses. The induction of IgA antibodies seems to be secondary to that of $\operatorname{IgG}$ antibodies in response to HPV intramuscular vaccination.

\section{P1.04 DEVELOPMENT OF AN ELISA-ASSAY FOR DETECTION OF IGA AND IGG AGAINST HUMAN PAPILLOMA VIRUS}

${ }^{1}$ Ana Paula Ferreira Costa, ${ }^{1}$ Paula Renata Lima Machado, ${ }^{1}$ Janaina Oliveira Crispim, ${ }^{2} J o s e ́$ Eleutério Júnior, ${ }^{3}$ Paulo César Giraldo, ${ }^{1}$ Ana Katherine Gonçalves anaina Oliveira Crispim. ${ }^{1}$ Federal University of Rio Grande do Norte, Natal - RN, Brazil; ${ }^{2}$ Federal University of Ceará, Fortaleza - CE, Brazil; ${ }^{3}$ University of Campinas, Campinas - SP, Brazil

10.1136/sextrans-2017-053264.112

Introduction The interest in human papilloma virus (HPV) seropositivity has increased considerably since HPV vaccines have become available worldwide. The aim of this study was to assess the performance of enzyme-linked immunosorbent assay (ELISA) in analysing serum samples provided from women with and without genital DNA-HPV infection confirmed by polymerase chain reaction (PCR), for detection of specific antibodies of the isotypes $\operatorname{IgG}$ and $\operatorname{IgA}$ recognising HPV-16 and -18 , as well as virus-like particles (VLPs).

Methods 50 sexually active female patients between 18 and 35 years of age from the outpatient clinic at the university hospital were enrolled. In order to test them, positive controls were obtained from patients with HPV-induced lesions and who were DNA-HPV positive confirmed by PCR. A specific assay was used to identify antibodies to HPV VLPs by ELISA. The samples were divided into HPV positive and negative, and an ELISA detecting IgA and IgG anti-HPV-VLP was carried out.

Results The effectiveness of ELISA and the kappa (k) index was obtained from the values entered in the receiver operating characteristic (ROC) curves for IgG and IgA. IgG-VLP-HPV-16 showed a good correlation between ELISA and PCR $(k=0.75)$, and IgG-VLP-HPV-18 showed a very good correlation between ELISA and PCR $(k=0.84)$. While the IgA antibody correlation was also positive, although weaker, IgA-VLP-HPV-16 was moderate $(k=0.45)$ and IgA-VLP-HPV-18 good $(k=0.66)$. The efficacy of the assay concerning IgG was: sensitivity, specificity, and accuracy were $82.3 \%, 92 \%$, and $88 \%$ to IgG-VLP-HPV16 , and $100 \%, 92 \%$, and $94 \%$ to IgG-VLP-HPV-18. The assay concerning IgA was: sensitivity, specificity, and accuracy were $64.7 \%, 80 \%$, and $73.8 \%$ to IgA-VLP-HPV-16, and $100 \%$, $80 \%$, and $84.8 \%$ to IgA-VLP-HPV-18.

Conclusion IgG and IgA antibodies against HPV-16 and -18 can be detected in unvaccinated individuals by using the VLP that serve as the basis for bivalent HPV vaccine. The values for ELISA assays and the values found for IgG correlate good/ very good with HPV-16/18 detected by PCR.

\section{P1.05 CURRENT USE AND PERCEIVED OBSTACLES TO USE OF POINT-OF-CARE TESTS IN SUB-SAHARAN AFRICA}

${ }^{1}$ Anne Rompalo, ${ }^{2}$ Ruth Kikonyogo, ${ }^{1}$ Yu-Hsiang Hsieh, ${ }^{1}$ Yukari Manabe, ${ }^{1}$ Charlotte Gaydos, ${ }^{3}$ Rosalind Parkes-Ratanshi. 'Johns Hopkins School of Medicine, United Sates; ${ }^{2}$ Infectious Diseases Institute, Makerere University, Uganda; ${ }^{3}$ Cambridge Institute of Public Health, UK

\subsection{6/sextrans-2017-053264.113}

Introduction Sexually transmitted infections (STIs) continue to be a significant global public health issue. Rapid, inexpensive and easy-to-use point of care tests (POCTs) for syphilis, HIV, and trichomonas have become available and WHO pre-qualified. We surveyed public health workers from Africa to determine current STI POCT practices.

Methods From March to October 2016, we invited 7584 healthcare workers (HCW) to participate in an email survey using Qualtrics software. Email addresses were obtained from the Ministry of Health and a database of training attendees at the Infectious Diseases Institute over the last 6 years.

Results 555 participants (50\% male) took the online survey. 91\% were from Uganda. Most $(n=449,81 \%)$ were clinicians including 336 (61\%) nurses and 113 (20\%) clinical officers of Level III/IV health centres (40\%). Among 449 clinician respondents, $312(69 \%)$ reported seeing HIV/general patients and $309(70 \%)$ diagnose at least one patient with an STI daily. Of 555 participants, common POCTs used were pregnancy test (74\%), urine dipstick (71\%), syphilis rapid test (66\%) and Gram's stain (53\%). Clinicians reported that $86 \%$ of POCTs were performed by HCWs and 54\% read the test while the patient was present. Most clinicians sent blood specimens for HIV (79\%) and syphilis (64\%) confirmation but only $18 \%$ routinely sent cultures for gonorrhoea. The majority of clinicians (74\%) practiced syndromic diagnosis for STDs/ HIV. Among all, lack of availability of POCTs (43\%), increased patient wait time (25\%), and lack of training to perform the test (21\%) were leading barriers to scale up POCTs. Comparing those who use syphilis POCTs to those who do not, a interruption of work flow was the greatest barrier identified $(20 \%$ versus $12 \%, \mathrm{p}=0.042) .60 \%$ of clinician participants felt that having increased access to POCTs for STIs would be useful.

Conclusion Increase of POCT availability and training could improve uptake of POCTs for STIs in Uganda and decrease the need for syndromic management which may lead to overtreatment and emergence of antibiotic resistance.

\section{P1.06 IN SILICO MULTILOCUS SEQUENCE TYPING OF CHLAMYDIA TRACHOMATIS PLASMIDS SHOWS CLUSTERING OF ISOLATES ACCORDING TO THE DISEASE RELATED BIOVARS}

${ }^{1}$ Bart Versteeg, ${ }^{1}$ Sylvia Bruisten, ${ }^{2}$ Odile Harrison, ${ }^{2}$ Keith Jolley, ${ }^{2}$ Martin Maiden, ${ }^{3}$ Arie Van Der Ende, ${ }^{3}$ Yvonne Pannenkoek. 'Public Health Service Amsterdam, Amsterdam, The Netherlands; ${ }^{2}$ University of Oxford, Oxford, UK; ${ }^{3}$ Academic Medical Centre, Amsterdam, The Netherlands

\subsection{6/sextrans-2017-053264.114}

Introduction Nucleotide sequencing of the ompA gene, encoding the outer membrane protein MOMP, divides C. trachomatis into 15 main genovars comprising three biovars associated 